\title{
Automatic Evaluation of Spatial Representations for Complex Robotic Arms Manipulations
}

\author{
Philippe Fournier-Viger ${ }^{1}$, Roger Nkambou ${ }^{1}$, André Mayers ${ }^{2}$ and Daniel Dubois ${ }^{1}$ \\ ${ }^{I}$ Department of Computer Science, University of Quebec at Montreal \\ ${ }^{2}$ Department of Computer Science, University of Sherbrooke \\ Fournier-Viger.Philippe@courrier.uqam.ca
}

\begin{abstract}
This paper describes how a knowledge model allows training software to evaluate spatial cognitive maps and provide tailored assistance.
\end{abstract}

\section{Introduction}

Many tasks involve relying on complex spatial representations. One such task is the manipulation of the CandarmII arm (CA) on the international space station (ISS). The CA is a robotic arm with seven degrees of freedom. Handling it is a demanding duty since astronauts who control it have a view of the environment rendered by three monitors. Each one show the view usually obtained from a single camera at a time among about ten cameras mounted at different locations on the ISS and on the CA. Guiding the CA requires several skills such as selecting cameras for a situation, visualizing in 3D an environment perceived in 2D and selecting efficient sequences of manipulations. Astronauts follow a rigorous protocol that comprises many steps, because one mistake can engender terrible consequences. To achieve the task, astronauts need to build spatial representations and to visualize them in a dynamic setting. Our team is working on RomanTutor [4], a software program for training to manipulate the $\mathrm{CA}$. The GUI reproduces part of CA's control panel. The task of interest in this paper is moving the $\mathrm{CA}$ from one configuration to another, according to the protocol. The aim of the work presented here is to describe the relevant cognitive processes of learners so that the software can follow their reasoning to grant a tailored assistance.

\section{Spatial cognition}

Since more than fifty years, many researchers have been interested in the knowledge involved in spatial reasoning. In the light of the researches carried out during the last decades, there is no doubt that humans rely on egocentric representations (which encode the space from the person's perspective) and allocentric "representations (independent of any point of view) [6]. An egocentric representation describes a route to follow to go from one place to another, and it is composed of an ordered set of stimuli/response associations [7]. Usually, this knowledge is gained through experience, but it can also be acquired directly (for instance, from textual route instructions). Route navigation is very inflexible and leaves little room for deviation. Indeed, choosing correct directions with landmarks strongly depends on the relative position of a person to landmarks. Consequently, a path deviation can easily disturb the achievement of the whole navigation task. An incorrect encoding or recall can also compromise seriously the attainment of the goal. Egocentric representations may be sufficient to travel through an environment, but they are inadequate to perform complex reasoning [7]. For reasoning that requires inference, humans build cognitive maps that do not preserve measurements but keep the main relationships between elements. These representations do not encode any perspective but makes it possible to adopt several perspectives. Cognitive maps are also prone to encoding or recall errors. But, it is generally easier to recover from an error, when relying on cognitive maps than on an egocentric representation. Tversky [7] indicates that a parallel can be drawn between cognitive maps and the semantic memory. Since these latter are key to complex spatial reasoning, tutoring software that diagnose and teach complex spatial reasoning should possess the ability to evaluate semantic knowledge.

\section{The cognitive model}

Our model for describing cognitive processes [2] is inspired by the Miace [5] cognitive theory, which attempt to model the human process of knowledge acquisition. As [5], we distinguish semantic knowledge, procedural knowledge and episodic knowledge. This paper does not explain the episodic memory part of our model since it is not central to the discussion, here. The semantic memory contains descriptive knowledge. Our model regards semantic 
knowledge as concepts taken in the broad sense. According to Halford \& al. [3], humans consider up to four concept instances simultaneously (four dimensions) in the achievement of a task. However, the human cognitive architecture is able to group several of them to handle them as one, in the form of a vector of concepts [3]. We call described concepts these concepts, in contrast with primitive concepts that are syntactically indecomposable. For example, " $3+5$ " is a decomposable representation of the symbol " 8 ", an undividable representation with the same semantic. The concept " $3+5$ " represents the sum of the number three and the number five, two primitive concepts. The addition operator " + " is also a primitive concept. In this way, the semantic of a described concept is given by the semantics of its components. The procedural memory encodes the knowledge of how to attain goals automatically by manipulating semantic knowledge. It is composed of procedures which fires one at a time according to the current state of the cognitive architecture [1]. Contrary to semantic knowledge, activating a procedure does not require attention. For example, when someone add automatically " $3+5$ " to obtain the sum " 8 ", the person does not recall the knowledge explicitly. As [5], we differentiate primitive and complex procedures. Whereas the first are seen as atomic actions, activating a complex procedure instantiates a set of goals, to be achieved either by a complex procedure or a primitive procedure. We consider goals as a special type of semantic knowledge. Goals are intentions such as the goal to solve a mathematical equation or to draw a triangle [5]. A goal is realized by means of procedure(s) execution. There can be many correct and incorrect ways (procedures) to achieve a goal. In our model, a goal has zero or more concept instances components, which are the object of the goal, and are determined by the complex procedure that instantiated the goal. Our model describes knowledge entities according to sets of slots. Concepts are encoded according to six slots. The "Identifier" slot is a character string used as a unique reference to the concept. The "Metadata" slot provides general metadata about the concept. The "Goals" slot contains a goals prototypes list; it provides information about goals that students could have and which use the concept. "Constructors" specifies the identifier of procedures that can create an instance of this concept. "Components" is only significant for described concepts. It indicates, for each concept component, its concept type. Lastly, "Teaching" points to some didactic resources to teach the concept. Goals have six slots. "Skill" specifies the necessary skill to accomplish the goal, "Identifier" is a unique name for the goal, "Metadata" describes the goal metadata, "Parameters" indicates the types of the goal parameters, "Procedures" contains a set of procedures that can be used to achieve the goal, and "Didactic-Strategies" suggests strategies to teach how to achieve that goal. Ten slots describe procedures. The "Metadata" and "Identifier" slots are the same as for concepts/goals. "Goal" indicates the goal for which the procedure was defined. "Parameters" specifies the concepts type of the arguments. For primitive procedures, "Method" points to a Java method that executes an atomic action. For complex procedures "Script" indicates a set of goals to be achieved. "Validity" indicates if the procedure always gives the expected result. "Diagnosis-Solution" contains data that indicate for each diagnosis, the suitable teaching strategy to be adopted. Finally, "Didactic-Resources" points to additional resources to teach the procedure.

Though the model was successfully employed in a tutoring system [2], it lays the emphasis on procedural knowledge learning and offers less support for semantic knowledge learning. The reason is that there is no structure for modeling the retrieval of knowledge from semantic memory, a key feature of many cognitive theories. Evaluating semantic general knowledge is essential for diagnosing and teaching spatial reasoning. To address this issue we define general knowledge, the semantic knowledge memorized or acquired through experience that is true in all situations of a curriculum. To be used properly, general knowledge must (1) be properly acquired beforehand, (2) be recalled correctly and (3) be handled by valid procedures. A general knowledge is a special type of described concept, because to be useful it must represent a relation. We added three slots to described concepts. The "General" slot indicates if a concept is general. The "Valid" slot specifies the validity of the concept (true/false), and optionally the identifier of an equivalent valid concept. In addition, the "RetrievalComponents" slot specifies a set of concepts to be instantiated to create the concept components when the concept is instantiated. Table 1 presents a concept encoding the erroneous knowledge that the ISS module "MPLM" is connected below the ISS module "NODE2" (according to the ISSACS coordinate system). The "Valid" slot indicates an equivalent valid knowledge is the concept "MPLM_TopOf_NODE2".

We added a retrieval mechanism to connect procedures to the general knowledge in order to model the recall process. It works as the retrieval mechanism of ACT-R. We choose ACT-R, because our model is already based on that theory. A slot named "Retrievalrequest" is added to procedures, to express a retrieval request for a concept in semantic memory, by means of patterns, that specifies the identifier of a concept to be retrieved and zero or more restrictions on the value of 
its components. Table 2 shows the procedure "RecallCameraForGlobalView". Its execution requests the knowledge of a camera on the ISS that a global view of a location taken as parameter by the procedure. The "Retrieval-request" slot states that a concept of type "RGlobalView" is requested (a relation that state that a camera gives a global view of a place), and that its first component should be a place whose concept type match the type of the procedure parameter, and the second component need to be of type "Camera". A correct recall following the execution of this procedure will result in depositing an instance of "RGlobalView" in a one instance buffer. The instance will then be available to the next procedures to be executed.

Table 1. Partial definition of the concept "MPLM_Below_MPLM2".

\begin{tabular}{|l|l|}
\hline Identifier & MPLM_Below_Node2 \\
\hline Components & Module, Module \\
\hline RetrievalComponents & MPLM, Node2 \\
\hline General & True \\
\hline Valid & $\begin{array}{l}\text { false } \\
\text { (true : MPLM_TopOf_Node2) }\end{array}$ \\
\hline
\end{tabular}

Table 2. Partial definition of the procedure "RecallCameraForGlobalView".

\begin{tabular}{|l|l|}
\hline Identifier & RGlobalView \\
\hline Goal & GoalRecallCameraForGlobalView \\
\hline Parameters & (ConceptPlace: $p$ ) \\
\hline $\begin{array}{l}\text { Retrieval- } \\
\text { request }\end{array}$ & $\begin{array}{l}\text { ID: RelationshipCameraGlobalView A1: } \\
\text { ConceptPlace A2: ConceptCamera }\end{array}$ \\
\hline
\end{tabular}

We have modelled the knowledge for the task of moving the CA from one position to another. We discretized the $3 \mathrm{D}$ space into $3 \mathrm{D}$ sub spaces (SS). The spatial knowledge is encoded as relations (described concepts) such as a camera can see a SS or an ISS module, a SS comprise an ISS module, a SS or ISS module is next to another, or a camera is attached to an ISS module. Moving the arm is modelled as a loop where one must recall a set of cameras for viewing the SSs containing the arm, select the cameras, adjust their parameters, retrieves a sequence of SSs to go from the current SS to the goal, and then move to the next SS. RomanTutor detects all the actions like camera changes and entering or leaving an SS. Describing the task in finer details (like choosing the right joint(s) to move to the next SS) is part of our current work.

The model provides two ways for evaluating general knowledge. First, the tutoring system can test general knowledge directly with questions. For example, RomanTutor may ask to name the closest modules to a given module or to ask to select the best cameras for viewing one or more modules. Second, general knowledge can be evaluated through problemsolving exercises. The result of each procedure makes it possible to infer through backward reasoning if a general knowledge was recalled (the result of the procedure allow deducing the retrieval buffer content). If the learner uses procedures to retrieve a valid knowledge several times, the system can suppose that the learner possesses that general knowledge. Similarly, the system tracks the use of erroneous knowledge. After building a portrait of the procedural and semantic knowledge of a learner, it generates tailored demonstrations, exercises and questions that involve the knowledge to be trained for.

\section{Conclusion and Further Work}

We presented a model for describing domain knowledge in tutoring systems to evaluate and teach semantic knowledge that learners should possesses. Because the model link semantic knowledge retrieval to procedural knowledge, evaluation of the semantic knowledge can also be achieved through problemsolving observation. Virtual tutors based on our model should be able to generate better feed-back, because they know how the recalled semantic knowledge is connected to procedures. Moreover, this paper showed how the model can be used to support spatial reasoning. Evaluating the effectiveness of our approach with humans is part of our future work.

\section{References}

[1] Anderson, J. R. (1993). Rules of the Mind, Hillsdale, NJ: Erlbaum.

[2] Fournier-Viger P., Najjar, M., Mayers, A. \& Nkambou, R. (2006). "A Cognitive and Logic based Model for Building Glass-box Learning Objects", Interdisciplinary Journal of Knowledge and Learning Objects, 2, pp. 77-94.

[3] Halford, G.S., Baker, R., McCredden, J.E. \& Bain, J.D. (2005), "How many variables can humans process?", Psychological Science, 16(1), pp. 70-76.

[4] Kabanza F., Nkambou R. and Belghith K. "Path-Planning for Autonomous Training on Robot Manipulators in Space". Proceedings of IJCAI 2005.

[5] Mayers A., Lefebvre B \& Frasson C. (2001). "Miace: A Human Cognitive Architecture". Sigcue outlook. 27(2), pp. 61-77.

[6] Nadel, L. \& Hardt, O. (2004) "The Spatial Brain", Neuropsychology, 18(3), pp. 473-476.

[7] Tversky, B. (1993). "Cognitive Maps, Cognitive Collages, and Spatial Mental Models", Proceedings COSIT'93, pp.14-24.

Acknowledgments. The authors thank the Canadian Space Agency and the Natural Sciences and Engineering Research Council for their logistic and financial support, and members of the GDAC/PLANIART involved in the development of RomanTutor. 